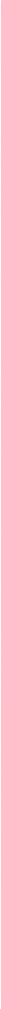

\title{
Was tun, wenn MFA die Corona-Impfung ablehnen?
}

Angestellte im Gesundheitswesen werden derzeit gegen das Corona-Virus geimpft. Sagen medizinische Fachangestellte "Nein" zur Corona-Impfung, sind die Arbeitgeber an der Reihe, Lösungen zu finden. Eine Kündigung muss immer das letzte Mittel bleiben.

Bedenken gegen die Corona-Impfung sind auch bei medizinischem Personal zu finden. Ärzten, deren medizinische Fachangestellte (MFA) sich trotz eines Impfangebots nicht gegen das Virus impfen lassen wollen, sind arbeitsrechtlich allerdings die Hände gebunden. „Im Bereich des Arbeitsschutzes ist eine Immunisierung gegenüber Infektionskrankheiten nach derzeitiger Rechtslage für die Beschäftigten freiwillig“, erklärt die Berufsgenossenschaft für Gesundheitsdienst und Wohlfahrtspflege $(B G W)$.

Auch Rechtsanwalt Dr. Florian Langenbucher sieht die Situation ähnlich: „Der Arbeitgeber kann nicht unmittelbar anordnen, dass eine Impfung stattfindet.“ Entsprechend schwierig sei es, wegen Nicht-Impfens eine Kündigung auszusprechen: Eine Beendigung des Arbeitsverhältnisses sei immer das letzte Mittel, betont Langenbucher.

Erstveröffentlichung in URO-NEWS (2021) 25(4):48-49

best practice onkologie $2021 \cdot 16$ (6): 300-302

https://doi.org/10.1007/s11654-021-00309-9

Online publiziert: 21. April 2021

(c) Springer Medizin Verlag GmbH, ein Teil von Springer Nature 2021
Wann kommt die Kündigung überhaupt infrage?

In Bereichen mit hoher Infektionsgefahr, etwa mit direktem Patientenkontakt, könnte das Risiko, das von nicht geimpften Mitarbeitern ausgeht, auch Auswirkungen auf das Arbeitsverhältnis haben: „In diesem Fall ist es durchaus denkbar, dass der Ausspruch einer ordentlichen personenbedingten Kündigung infrage kommt", so der Rechtsexperte. Aber auch das sei nicht ohne Weiteres möglich: Vor einer Beendigungskündigung müsse der Arbeitgeber im Einzelfall prüfen, ob eine Umgestaltung des Arbeitsplatzes, eine Versetzung oder eine Änderungskündigung möglich sei.

Nur, wenn durch Umgestaltungs- oder Versetzungsmaßnahmen die hohe Infektionsgefahr nicht eingedämmt werden kann, könnte also eine Kündigung im Einzelfall möglich sein. Persönliche Schutzkleidung könnte hier aber bereits notwendige Abhilfe schaffen. „Auch wenn die COVID-19-Impfungen einen Meilenstein in der Pandemiebekämpfung bedeuten, sind weiterhin Infektions- und Arbeitsschutzmaßnahmen umzusetzen", betont die BGW.

Wenn sich einzelne MFA nicht impfen lassen wollen, gelte es laut Verband medizinischer Fachberufe $(\mathrm{VmF})$ zuerst den Dialog zu suchen: „Ärzt ${ }^{*}$ innen und MFA sollten miteinander reden, denn häufig fehlt es an Aufklärung über Wirkung und Nebenwirkungen der Impfung gegen COVID-19. Dabei sollte auch über die verschiedenen Impfstoffe gesprochen werden. Im Übrigen können nur informierte und überzeugte MFA die Patient ${ }^{\star}$ innen bei den geplanten Impfungen in den Arztpraxen 
für die Impfung gegen COVID-19 informieren. Die Investition in die Aufklärung lohnt sich daher doppelt.“

\section{Versetzung ist eine Option}

Sollte eine MFA ihre Skepsis nicht ablegen, müssten Lösungen für das gesamte Team gesucht werden: Man könne wie bei MFA, die selbst der COVID-19-Risikogruppe angehören, eine Versetzung in andere Arbeitsbereiche oder mobiles Arbeiten ermöglichen.

Der Verband spricht sich insgesamt gegen eine CoronaImpfpflicht aus. „Ob man aufgrund der besonderen Situation der Pandemie möglicherweise aus der allgemeinen arbeitsrechtlichen Treuepflicht eine Impfpflicht herleiten kann, werden im Einzelfall die Arbeitsgerichte entscheiden müssen. Dabei ist immer das Interesse der Arbeitgeberseite an der Impfung der Mitarbeiter*innen gegen das Interesse dieser Mitarbeiter*innen an der Wahrung ihrer/seiner Intimsphäre und körperlichen Unversehrtheit abzuwägen“, betont der VmF.

Margarethe Urbanek

Hier steht eine Anzeige.

Springer 
Hier steht eine Anzeige.

Springer 